\title{
Exploratory analysis of deficits in DRL performance induced by medial and lateral fornix damage
}

\author{
FRED H. GAGE and SELBY H. EVANS \\ Texas Christian University, Fort Worth, Texas 76129
}

\begin{abstract}
A reanalysis was conducted of DRL performance in rats with medial or lateral fornix damage. The reanalysis used multivariate procedures proven effective in analyzing DRL performance in normal rats. The original analysis found a deficit after medial fornix damage. The reanalysis of the data revealed more specifically that the deficit following medial fornix lesions was in the animals' failure to suppress short-latency responses. On the other hand, animals with lateral fornix damage demonstrated a slight deficit in their ability to localize responding to the earliest period in which reinforcement was available. Alternative interpretations of these data are presented in terms of response inhibition and discrimination deficits.
\end{abstract}

Differential reinforcement of low rate schedule (DRL) is in common use as an assay of animal behavior preceding and following a variety of physiological manipulations of the limbic system (Numan, 1979). At least four separate measures of DRL performance have been used to assess behavior: (1) response rate, the number of responses per unit time; (2) reinforcement rate, the number of reinforcements per unit time; (3) efficiency, the reinforcement rate divided by the response rate; (4) IRT/OP, the estimated conditional probability of responding in any particular time interval, given an opportunity to respond in that interval (Anger, 1956).

The above measures have often been used to represent the performance of the animals in terms of "response inhibition" or "temporal discrimination" (Johnson, Olton, Gage, \& Jenko, 1977). In this context, inhibition refers to the ability of the animal to inhibit or prevent a response before the timing interval has ended. In contrast, temporal discrimination refers to timing behavior and to the ability of the animal to discriminate the passage of the required time interval since the last barpress.

Principal components analysis of IRT/OP data obtained during acquisition by intact animals identified two independent components of variance (Gage, Evans, \& Olton, 1979). One, associated with responses in unreinforced intervals, was well represented by measures such as reinforcement rate or efficiency. The other component, which might be considered to represent temporal discrimination, was associated with responding in the first reinforced interval. We now report a reanalysis of DRL performance after medial and lateral fornix damage.

Correspondence should be sent to Fred H. Gage, Department of Psychology, Texas Christian University, Fort Worth, Texas 76129 .
The fornix-fimbria distributes its fibers heterogeneously to the septal area and hypothalamus from the hippocampal formation (HF) and to the HF from the septal area and more caudal brain areas (Siegel, Edinger, \& Ohgami, 1974; Siegel \& Tassoni, 1971). Specifically, reciprocal connections exist between the dorsal HF and the midline septal nuclei via the medial components of the fornix. In addition, reciprocal connections exist between the ventral HF via the lateral fimbria fornix to the lateral septal nuclei (Meibach \& Siegel, 1977).

In the present study, this distinctive, although somewhat controversial, circuitry in the fornix was used to separate dorsal HF from ventral deficits by comparing performance on a DRL task in animals following damage to either medial or lateral fornix. Given the evidence for reciprocal connections between septal nuclei and the hippocampus, via the fornix, similar deficits to those observed from the medial fornix lesion would be anticipated following medial septal damage and dorsal hippocampal damage. In addition, similar deficits to those observed following lateral fornix lesions would be expected following lateral septal damage and ventral hippocampal damage. These speculations, of course, require experimental verification, even though the hypothesis follows from the anatomical reports.

The original analysis of these data found an initial decline in efficiency and an increase in responding during early IRT/OP intervals following medial fornix lesions, with no change initially following lateral fornix lesions (Johnson et al., 1977). Our reanalysis sought to characterize the medial fornix effect in terms of the newly identified components of the IRT/OP data and to determine whether an initial effect of lateral fornix damage could be identified by examining both components or by other applications of multivariate methods. 


\section{METHOD}

\section{Subjects}

The subjects were male Sprague-Dawley (Camm) albino rats, 90-120 days old at the beginning of the experiment. All animals were housed individually, with ad-lib access to water.

\section{Behavioral Testing}

All animals were reduced to $85 \%$ of their ad-lib body weights by restriction of food intake over a period of 5 days. They were maintained at this level throughout behavioral testing by provision of the appropriate amount of food in their home cages approximately $1 \mathrm{~h}$ after each day's test. All behavioral test sessions were $30 \mathrm{~min}$ in duration. For the first 2 days of operant testing, animals were shaped to leverpress by the placement of food powder on the lever; reinforcement was provided on a continuous schedule. On the third and subsequent days, the DRL 20-sec schedule was used. Preoperative DRL-20 testing took place daily for at least 30 days; at the end of this period, the performance of most animals had stabilized.

After 30 days of DRL 20, the preoperative testing of the individual animal was discontinued if both of the following criteria were met: (1) the animal exhibited a daily efficiency score (number of reinforced leverpresses in that session divided by the total number of leverpresses times 100) on at least $20 \%$ for 5 consecutive days, and (2) the performance of the animal was relatively stable for these 5 days so that the standard deviation of the five efficiency scores was less than 10. Animals attaining these criteria were divided into the various surgical and unoperated control groups; an attempt was made to match all groups on the basis of preoperative efficiency scores. Animals that failed to reach criteria (1) and (2) within 55 days were dropped from the experiment.

Following surgery, the animals were allowed 2 weeks for recovery. During the first week of this period, food was available ad lib. During the second week of recovery, the animals were once again reduced to $85 \%$ of their preoperative ad-lib body weights by a food-deprivation schedule. Postoperative DRL testing, identical to preoperative testing, was begun and continued for at least five sessions. For specific details of apparatus, surgery, and histology, see Johnson et al. (1977).

\section{Data Analysis}

Performance was analyzed in two blocks of 5 days each, one immediately before the operation and the second immediately after. The IRT/OP measure was obtained from the formula discussed by Anger (1956). Twelve counting bins of $4 \mathrm{sec}$ were used, but the results for only the first seven were retained for analysis.

As noted in the previous report, the IRT/OP measure is not necessarily the best choice for multivariate analysis of DRL data because the divisor for any period except the first is determined by what happened in the shorter periods. This circumstance may introduce artifactual correlations. We use the IRT/OP measure here to maintain continuity with previous research.

The analytic method was exploratory data analysis in the sense characterized by Tukey $(1969,1977)$. Exploratory data analysis relates to confirmatory data analysis in much the same way that detective work relates to court trial. Exploratory data analysis is directed toward the identification of relationships that may merit testing as hypotheses in subsequent research and toward the specification of analytic procedures that are most likely to support successful inquiry.

Since exploratory data analysis seeks to identify promising relationships within a data set, it cannot properly apply standard statistical tests. These tests are, for the most part, designed to test hypotheses formulated without influence from the data set to be analyzed. Exploratory data analysis may thus risk increased exposure to misdirection from random variation. We suggest three rules of practice that help to control that risk. First, the analysis and the conclusions need to be guided by the reliability of the data, as assessed in the course of the exploratory analysis. Second, con- clusions ought to be supported by internal confirmation from more than one piece of evidence from the data. Third, results should be regarded as provisional, requiring replication (in which confirmatory data analysis would be appropriate).

When multiple dependent measures are under study, exploratory data analysis is necessarily concerned with finding ways to summarize these measures, with identifying summary measures that are most sensitive to the experimental manipulations, and with describing the treatment effects in terms of these summary measures. Principal components analysis was used in the present study to assist in summarizing the multivariate data. Although principal components analysis is often treated as a form of factor analysis, it is based on different assumptions (Lee \& Comrey, 1979) and can properly stand on its own as a method of identifying a reduced set of dimensions that provide a parsimonious summary of a set of intercorrelated variables (Tatsuoka, 1971).

\section{RESULTS}

The report of results is organized in terms of the questions asked of the data.

(1) Are the preoperative subjects homogeneous in their DRL performance?

An inquiry into this question was specifically indicated by the observation in the original study that not all the rats reached the criteria described earlier. The rats available for the present analysis had met those criteria, but it is possible for subjects to satisfy univariate criteria and still be substantially different from the main body of subjects in a multivariate sense. Thus, a multivariate inquiry called for a reexamination of the question of homogeneity, this time in terms of the multivariate data. More generally, however, any exploratory analysis of multivariate experimental data can probably benefit from an initial inquiry into the homogeneity of the subjects if the paradigm provides appropriate data.

This question can be addressed by a Q-factor form of principal components analysis. In this form, the variables play the role of subjects and vice versa. (In the present study, all the variables are measured on the same scale; if the variables are not measured on the same scale, additional considerations of scale and scale adjustments are required for this analysis.) The result of the analysis is a set of independent components of variance that together account for the correlation matrix taken between subjects. A homogeneous group of subjects should result in a single principal component accounting for most of the variance.

The analysis was done on the data from the last preoperative week. About $75 \%$ of the variance was accounted for by the first principal component. Since all of the measures were on the same scale, this value provides an estimate of the proportion of reliable variance in the data set, taken across all subjects and variables. Most subjects correlated above .7 with this component. Four subjects did not and were dropped from further analysis.

The choice of cutting point for the elimination of outliers was based on inspection of the distribution 
of correlations. The four subjects that were dropped had correlations with the first principle component ranging from -.42 to .56 . Among those subjects that were retained, the four lowest correlations were .67 , $.72, .73$, and .73. A cutting point near .7 is also reasonable on a priori grounds. Such a correlation means that the subject has about $50 \%$ of its variance in common with the rest of the subjects. It seems unlikely that one would wish to discard subjects that met that criterion, regardless of the shape of the distribution. Furthermore, if the data demanded a much more lenient criterion (because the .7 criterion would discard too many subjects), the investigator might want to consider whether there is enough reliable variance to justify the effort of exploratory analysis.

(2) Does the analysis of the control subjects in this study replicate the findings in our multivariate analysis (Gage et al., 1979) of DRL performance?

Table 1 shows results for the current and previous principal components analyses. The components found in the first study do replicate our previous findings. As in the previous study, the first principal component correlated primarily with early IRTsespecially 3 and 4 . The second principal component once again correlated with IRT6. There is a hint of a third component emerging in the present study. Note that the present results are based on experienced subjects, while the earlier results were based on the acquisition phase. The possibility of a third component, detectable only after the subjects have stabilized in performance, should be considered in any replication of this analysis.

In evaluating this replication, one should keep in mind that both data sets came from the same subjects and represent different phases of the same task. The primary conclusion to be drawn is that the principal components analysis can demonstrate replication of components in these kinds of data.

Table 1

Principal-Components Analysis of IRT/OP Data at End of Acquisition and Similar Analysis for Acquisition Phase

\begin{tabular}{ccccccc}
\hline & \multicolumn{5}{c}{ Correlation } \\
\cline { 2 - 4 } $\begin{array}{c}\text { IRT/OP } \\
\text { Period }\end{array}$ & \multicolumn{3}{c}{ Postacquisition } & & Acquisition \\
\cline { 2 - 4 } \cline { 5 - 6 } & F1 & F2 & F3 & & F1 & F2 \\
\hline 1 & -.44 & & .79 & .46 & -.64 \\
2 & .34 & -.41 & -.69 & .81 & -.43 \\
3 & .80 & -.46 & & .87 & \\
4 & .89 & -.23 & & .84 & .40 \\
5 & .66 & .22 & .32 & .61 & .67 \\
6 & .51 & .79 & & & .82 \\
7 & .30 & .63 & .20 & .30 & .53 \\
P & .36 & .22 & .18 & .53 & .24 \\
CP & .36 & .58 & .76 & .53 & .77 \\
\hline
\end{tabular}

Note-Postacquisition $N=41$; acquisition $N=150$ (from Gage, Evans, \& Olton, 1979). Correlations less than .17 were omitted. $P=$ proportion of variance accounted for; $C P=$ cumulative proportion of variance accounted for.

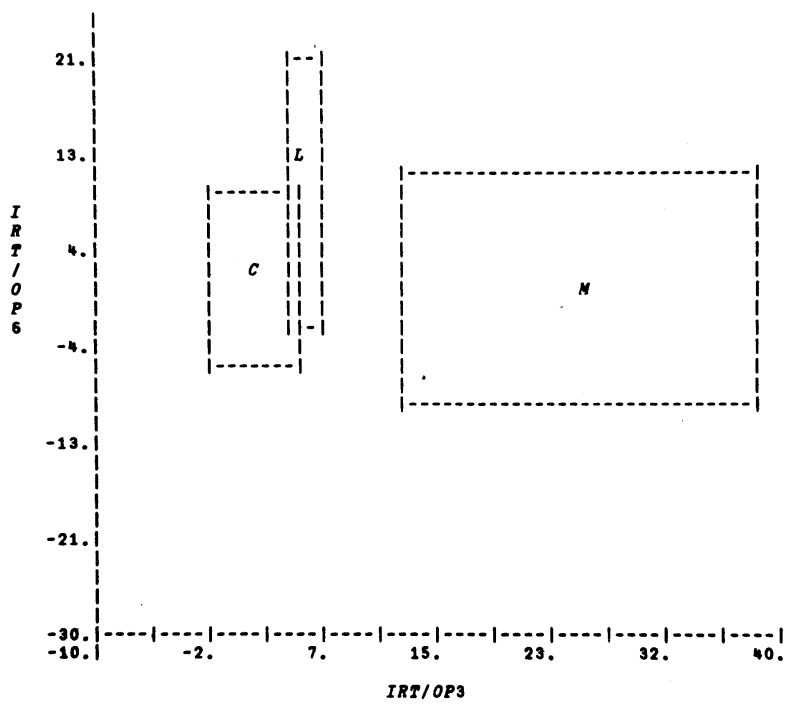

Figure 1. Location of experimental groups in the space representing the two components found in the previous study. Axes represent the change in performance from preoperative to postoperative (post minus pre) on IRT3, representing inhibition, and on IRT6, representing discrimination. The three groups are control (C), lateral fornix damage (L), and medial fornix damage (M). The boxes mark out regions 1 SD (within group) on either side of the group mean.

(3) Since the IRT/OP analysis shows two components in the data, how is performance on these two components affected by fornix lesions?

Change scores were obtained by subtracting each subject's mean IRT/OP scores on the last preoperative week from its scores on the first postoperative week of testing. The number of subjects available for this analysis was six for the control group, seven for the medial fornix group, and five for the lateral fornix group. (These numbers do not add up to the total $\mathrm{N}$ available for analysis in connection with Question 2 because the original study included several other treatment groups that were not used in the present investigation.)

Figure 1 shows the location of the three groups in the space defined by the two components. The boxes mark out \pm 1 SD (within group) on each axis and, for normally distributed data, would show the location on each axis of approximately two-thirds of the data points. The group with medial fornix lesions shows a substantial increase in responses in IRT3, as compared with the control group. The medial group also shows much greater variance than the control group.

On the IRT6 axis, the medial group completely overlaps the control group. Thus, the data rather clearly contradict the possibility that the medial lesion has an effect on responding in IRT6.

The group with lateral lesions partially overlaps the control group on both dimensions. Its location, however, is consistent with the possibility of a small treatment effect on both dimensions. 


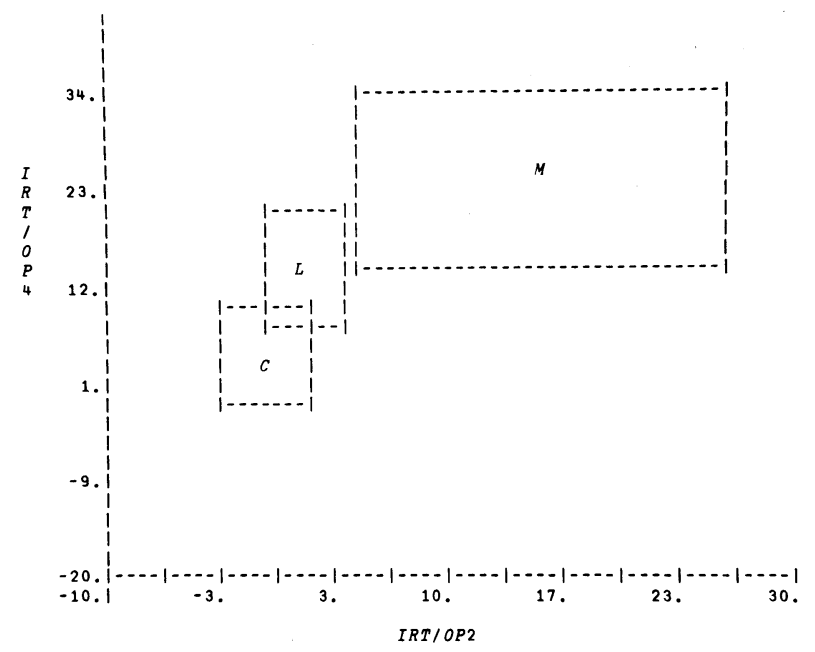

Figure 2. Location of experimental groups in the space defined by IRT/OP scores representing early and late portions of the "inhibition" period. The axes represent change in performance from preoperative to postoperative (post minus pre) on IRT2 and IRT4. The first reinforced period was IRT6. The three groups are control (C), lateral fornix damage (L), and medial fornix damage (M). The boxes mark out regions 1 SD (within group) on either side of the group mean. Note that the group with lateral fornix damage shows little effect on IRT2 but relatively greater effect on IRT4.

(4) What do the data indicate about the effect of lateral fornix lesions?

Examination of the data of the lateral fornix group for the other IRT/OP periods indicated a pattern similar to that noted above, particularly in IRT4 and IRT5. Figure 2 shows the relative locations of the three groups in the space defined by IRT2 and IRT4. The effect of the medial lesion is apparent in the displacement of that group from the control group on both axes. The lateral fornix group is also displaced from the control group on both axes and in the same direction as the medial group. The relatively stronger effect on IRT4 is seen in the substantial overlap of the lateral group with the medial group on that axis.

Since the lateral fornix group is consistently displaced in the same direction on the IRT/OP measures, we conclude that there probably was an effect of this treatment. Furthermore, the effect is most likely to be detectable in IRT/OP periods that are fairly close to the shortest interval on which reinforcement is available.

Efforts to replicate this phenomenon would need a larger number of subjects devoted to the lateral fornix group than was used in the present study. Such efforts should avoid combining both medial and lateral groups in the same analysis. The medial fornix lesion appears to be associated with a substantial increase in within-group variance. In an analysis of variance, this large within-group variance would result in an error term overestimating the error that applies to the lateral fornix and control groups. Thus, the inclusion of a medial fornix group would result in an unnecessary bias against finding an effect in the lateral group. The same advice, of course, could apply to the inclusion of a medial fornix group in any analysis with other experimental groups.

\section{DISCUSSION}

The results of the present study replicate well the finding of the previous study applying principal components analysis to DRL performance. Both analyses identified two separate summary measures representing distinct components of the IRT/OP data. One component corresponds to the suppression of short latency responses and can be represented by IRT3. The other corresponds to high response rates in the earliest period in which reinforcement is available and can be represented by IRT6 in the present data.

Following lesion in the medial fornix, a substantial increase in short latency responses was evident. The data indicated no change in rate of responding in IRT6. Animals with a lateral fornix lesion showed some indication of small increases in response rate in IRT6. These animals showed a consistent pattern of increases in response rates for IRT4 and IRT5, with diminishing effect for shorter IRT/OP intervals.

These results are subject to more than one interpretation. One could contrast the effects of medial and lateral fornix damage. The increase in response rate in the early IRT periods, resulting from medial fornix damage, could be regarded as loss of inhibition. The apparent increase in response in IRT/OP4, resulting from lateral fornix damage, could be interpreted as a weakening of temporal discrimination. On the other hand, both effects could be viewed as being on the same continuum, the effect of medial damage being a more profound loss of temporal discrimination or of inhibition.

The alternative interpretations probably cannot be resolved within the DRL paradigm because they do not have distinct implications there. Indeed, a temporal discrimination depends on inhibition, and a loss of inhibition is one way in which the discrimination could be abolished. A loss of inhibition, however, should affect other kinds of discrimination learning.

A paradigm that would be useful in distinguishing between the two alternatives could be based on mapping the characteristics of the DRL paradigm into a task that requires a nontemporal discrimination. For example, a brightness discrimination might be used with, say, six levels of illumination corresponding to the six key intervals in the present study. The different brightness levels would be presented in random order, but only the brightest would be reinforced. Responses in $\mathrm{S}^{\Delta}$ would be followed by time-out. Performance on this paradigm should not be disrupted by loss of temporal discrimination, if that is taken to mean accuracy in time estimation. A loss of ability to 
inhibit responses, on the other hand, should disrupt performance on this paradigm as much as in the DRL paradigm, assuming that the new task is "tuned" to make the discrimination approximately equal in difficulty.

Evidence is accumulating to indicate substantial functional differences between deficits induced by dorsal vs. ventral HF damage (Gage \& Olton, 1975; Olton, Walker, \& Gage, 1978) and medial vs. lateral septal damage (Poplowsky \& Johnson, 1973). A critical question to be addressed is whether these functional differences are quantitative or qualitative in nature-more specifically, whether the differences in observed behavioral deficits following damage to subareas of the septal hippocampal system are difficult in degree or in kind. To this end, the outline of the above proposed experiment, which potentially separates a response inhibition deficit from a temporal discrimination deficit, may aid in the solution to this question.

\section{REFERENCES}

Anger, D. Dependence of interresponse times upon the relative reinforcement of different interresponse times. Journal of Experimental Psychology, 1956, 52, 145-161.

Gage, F. H., Evans, S. H., \& Olton, D. S. Multivariate analyses of performance in a DRL paradigm. Animal Learning \& Behavior, 1979, 7, 323-327.

GAGE, F. H., \& Olton, D. S. Hippocampal influences on hyper- reactivity induced by septal lesions. Brain Research, 1975, 98, 311-325.

Johnson, C. T., Olton, D. S., Gage, F. H., \& Jenko, P. S. Hippocampus, hippocampal connections and DRL. Journal of Comparative and Physiological Psychology, 1977, 91, 508-522.

LeE, H. B., \& Comrey, A. L. Distortions in a commonly used factor analytic procedure. Multivariate Behavioral Research, 1979, 14, 301-321.

Meimbach, R. C., \& Siegel, A. Efferent connections of the hippocampal function in the rat. Brain Research, 1977, 124, 197-224.

Numan, R. Cortical-limbic mechanisms and response control: $\mathbf{A}$ theoretical review. Physiological Psychology, 1979, 6, 445-470.

Olton, D. S., Walker, J. A., \& Gage, F. H. Hippocampal connections and spatial discrimination. Brain Research, 1978, 98, 311-325.

Poplowsky, A., \& Johnson, D. A. Open field social behavior of rats following lateral and medial septal lesions. Physiology \& Behavior, 1973, 2, 845-854.

Siegel, A., Edinger, H., \& Ohgami, S. The topological organization of the hippocampal projection to the septal area: $\mathbf{A}$ comparative neuroanatomical analysis in the gerbil, rat, rabbit, and cat. Journal of Comparative Neurology, 1974, 157, 359-378.

Siegel, A., \& TAssoni, J. P. Differential efferent projections of the lateral and medial septal nuclei to the hippocampus in the cat. Brain, Behavior, and Evolution, 1971, 4, 201-219.

TAtsuoka, M. M. Multivariate analysis: Techniques for educational and psychological research. New York: Wiley, 1971.

TukEY, J. W. Analyzing data: Sanctification or detective work? American Psychologist, 1969, 24, 83-91.

TukEY, J. W. Exploratory data analysis. Reading, Mass: AddisonWesley, 1977.

(Received for publication June 6, 1980; revision accepted January 21, 1981.) 